\title{
A Patient with Primary Open-Angle Glaucoma with Re-Elevated Nocturnal Sitting Intraocular Pressure after Restarting Medical Therapy due to a Bleb Failure
}

\author{
Kenji Nakamoto, Naka Shiratori, Yusuke Nishio, \\ Shio Sugimoto, Yasuko Takano, Masashi Yamazaki, \\ Yutaro Tobita, Tsutomu Igarashi and Hiroshi Takahashi \\ Department of Ophthalmology, Nippon Medical School, Tokyo, Japan
}

\begin{abstract}
We describe the case of a primary open-angle glaucoma patient with re-elevated nocturnal sitting intraocular pressure (IOP) after restarting medical therapy due to a failing bleb. IOP was markedly higher than diurnal IOP during multiple-drug therapy in both eyes, but it did not increase in the left eye with a functional bleb without medical therapy after trabeculectomy with adjuvant mitomycin. However, nocturnal sitting IOP was re-elevated after restarting multiple-drug therapy due to a failing bleb, while diurnal IOP was maintained at a low level. (J Nippon Med Sch 2021; 88: 509-511)
\end{abstract}

Key words: 24-hour intraocular pressure, trabeculectomy, diurnal intraocular pressure variation, nocturnal intraocular pressure, glaucoma

\section{Introduction}

Glaucoma is a leading cause of visual function $\operatorname{loss}^{1,2}$. The only reliable treatment at present is the use of intraocular pressure (IOP) reduction therapy ${ }^{1,3}$. Antiglaucoma eye drops are generally started as the initial treatment for primary open-angle glaucoma (POAG) ${ }^{1}$. Prostaglandin F2 $\alpha$ analogs (PGF2 $\alpha$ ) have been commonly used as the first-line treatment for glaucoma ${ }^{1}$. PGF2 $\alpha$ has a 24-hour IOP-lowering effect in $\mathrm{POAG}^{3}$ and normal tension glaucoma ${ }^{4}$. However, with combination therapy of glaucoma eye drops, sitting IOP tends to increase during the nighttime in open-angle glaucoma ${ }^{5}$ and POAG $^{6,7}$. In contrast, trabeculectomy with adjunctive mitomycin C (TLE) can lower IOP much more than drug therapy during not only the daytime, but also the nighttime ${ }^{5}$. However, if clinic IOP re-elevates over the target IOP due to a failing bleb, glaucoma eye drops are generally re-started. In relation to both selective laser trabeculoplasty ${ }^{8,9}$ and trabeculotomy combined with sinusotomy ${ }^{10}$, there are some reports that nocturnal sitting IOP was significantly lower than preoperative sitting IOP, although there was no significant diurnal sitting IOP reduction. However, it is un- clear whether nocturnal sitting IOP re-elevates after restarting multiple-drug therapy due to a failing bleb after TLE while diurnal IOP maintains at a low level.

In the following report, we describe the case of a POAG patient with re-elevated nocturnal sitting IOP after restarting medical therapy due to a failing bleb.

\section{Case Report}

A 47-year-old woman with POAG was referred to our hospital for further examination and treatment due to rapid visual field deterioration in the left eye despite a well-controlled clinic IOP of approximately $12 \mathrm{mmHg}$. Her untreated clinic IOP was $18 \mathrm{mmHg}$ in both eyes according to her referral letter from a previous doctor. At her first visit, her preoperative spherical equivalents were $-0.5 \mathrm{D}$ in both eyes, and her corrected visual acuity was 20/20 in both eyes, with an IOP using Goldmann applanation tonometry (GAT) of $14 \mathrm{mmHg}$ in both eyes. Both her eyes were being treated with latanoprost ophthalmic solution $0.005 \%$ (latanoprost) eye drops once per day each night and timolol gel-forming solution $0.5 \%$ (timolol) eye drops once per day each morning. Slit lamp

Correspondence to Kenji Nakamoto, MD, Department of Ophthalmology, Nippon Medical School, 1-1-5 Sendagi, Bunkyo-ku,

Tokyo 113-8602, Japan

E-mail: user902850@aol.com

https://doi.org/10.1272/jnms.JNMS.2021_88-521

Journal Website (https://www.nms.ac.jp/sh/jnms/) 


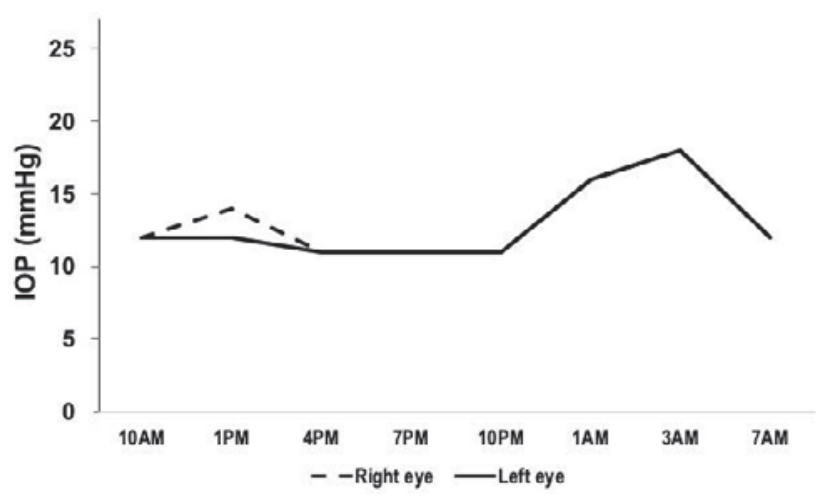

Fig. 1 Twenty-four-hour sitting IOP during multipledrug therapy

Nocturnal IOP at $1 \mathrm{AM}$ and $3 \mathrm{AM}$ were markedly higher than diurnal IOP during multiple-drug therapy in both eyes.

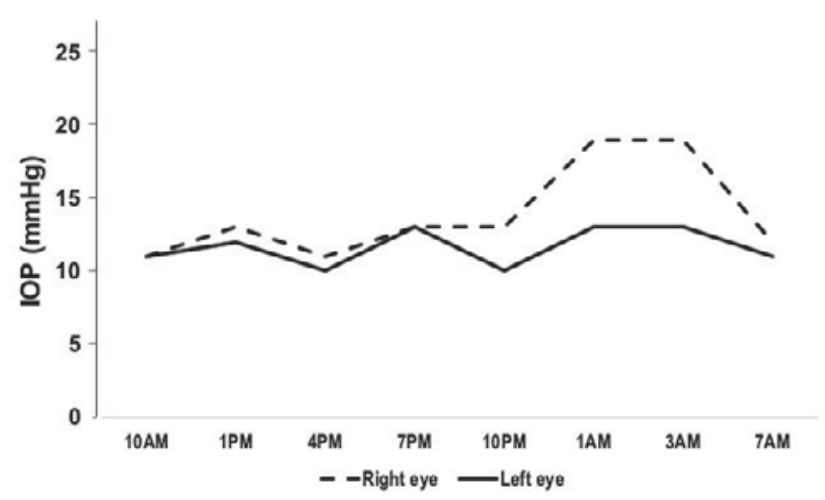

Fig. 2 Twenty-four-hour sitting IOP without medical therapy with a functional bleb after TLE

There was no nocturnal IOP elevation in the left eye, in contrast with the right eye. TLE: trabeculectomy with adjunctive mitomycin $\mathrm{C}$.

examination showed normal findings in both eyes. Gonioscopy showed open angles in both eyes. Fundus examination showed definite inferior temporal and superior temporal retinal nerve fiber defects and enlargement of optic disk cupping in both eyes. Humphrey perimetry program 30-2 performed by a previous doctor showed corresponding superior nasal and inferior nasal visual field defects in both eyes, and definite progression in the left eye. Because IOP elevation outside clinic hours was suspected, sitting IOP was measured at 10 AM, 1 PM, 4 PM, 7 PM, 10 PM, 1 AM, 3 AM, and 7 AM through 24-h periods by GAT during the medical therapy in the hospital (Fig. 1). Nocturnal IOPs at $1 \mathrm{AM}$ and $3 \mathrm{AM}$ were markedly higher than diurnal IOPs in both eyes. For the purpose of lowering 24-h IOP, TLE was performed in the left eye. At about 18 months after TLE, 24-h sitting IOP of the right eye during medical therapy (latanoprost, ti-

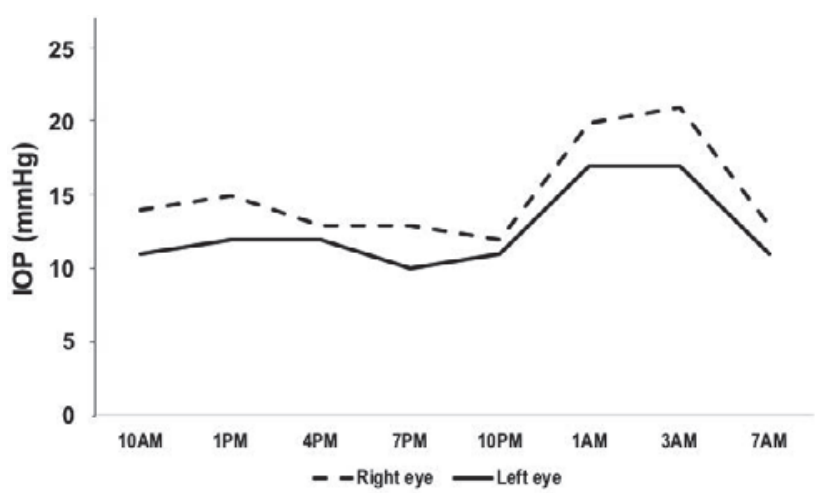

Fig. 3 Twenty-four-hour sitting IOP after restarting medical therapy due to a failing bleb

Nocturnal IOP was re-elevated at 1 AM and 3 AM in both eyes, although the IOP of the left eye is lower than that of the right eye at all 8 time points

molol, and brinzolamide $1 \%$ ophthalmic suspension twice per day each morning and night) and of the left eye without medical therapy were measured in the hospital (Fig. 2). There was no nocturnal IOP elevation in the left eye, in contrast with the right eye. However, left IOP increased to $15 \mathrm{mmHg}$ without glaucoma eye drops along with a failing bleb. Needle revisions of the bleb were performed twice in the left eye, but left IOP reelevated to $15-16 \mathrm{mmHg}$. Then, latanoprost and dorzolamide/timolol fixed combination (dorzolamide/timolol) twice per day each morning and night were instilled into both eyes. The clinic IOP in both eyes remained at 10-13 $\mathrm{mmHg}$ thereafter, but clinic IOP of the left eye was almost always lower than that of the right eye. At about 5 years after TLE, 24-h sitting IOP of both eyes was remeasured during medical therapy (latanoprost and dorzolamide/timolol) in the hospital. The nocturnal IOP was re-elevated at $1 \mathrm{AM}$ and $3 \mathrm{AM}$ in both eyes, although IOP of the left eye was lower than that of the right eye at all 8 time points (Fig. 3 ).

\section{Discussion}

IOP reduction treatment is the only evidence-based treatment for glaucoma at present. It is well-known that there are not a few patients whose sitting IOP measured outside clinic hours is high among those with rapid visual field defect progression despite low clinic IOP treated with anti-glaucoma eye drops ${ }^{10}$. In addition, nocturnal sitting IOP tends to be higher than diurnal sitting IOP during combination glaucoma drug therapy ${ }^{5-7}$. The mechanism remains unclear, but it may be due to a smaller pharmacological effect of almost all glaucoma drugs during the nighttime than during the daytime ${ }^{11,12}$. 
In contrast, successful TLE can lower 24-hour IOP much more than drug therapy throughout the day ${ }^{5}$, mainly because functional TLE has an aqueous pathway to the subconjunctival space ${ }^{13}$.

The present patient also had nocturnal sitting IOP elevation during combination glaucoma drug therapy. However, while the filtering bleb remained functional and medication was not needed, there was no nocturnal IOP elevation. In contrast, when the bleb function failed and multiple-drug therapy was again required, the nocturnal sitting IOP was re-elevated during the nighttime in the left eye, although the IOP of the left eye was lower than that of the right eye at all time points. We previously reported a significant positive correlation between mean 24-h sitting IOP and 24-h sitting IOP fluctuation in POAG patients without medication after $\mathrm{TLE}^{14}$. Thus, in order to maintain low sitting IOP throughout the day, keeping good bleb function and sitting IOP as low as possible without medication may be needed.

In summary, the present POAG patient developed reelevated nocturnal sitting IOPs after restarting medical therapy due to a failing bleb although the nocturnal elevation of IOP was suppressed with a functional bleb after TLE. This indicates that caution must be taken in patients with re-elevated nocturnal sitting IOP when restarting medical therapy after TLE even if clinic IOP is maintained at a low level.

Conflict of Interest: The authors have no conflicts of interest.

\section{References}

1. The Japan Glaucoma Society. Ryokunaisho shinryo gaidorain dai 4 han [Guidelines for Glaucoma 4th edition]. Nippon Ganka Gakkai Zashi [J Jpn Ophthalmol Soc]. 2018 Jan 10;122(1):3-53. Japanese.

2. Tham YC, Li X, Wong TY, Quigley HA, Aung T, Cheng CY. Global prevalence of glaucoma and projections of glaucoma burden through 2040: a systematic review and meta-analysis. Ophthalmology. 2014 Nov;121(11):2081-90.

3. Orzalesi N, Rossetti L, Invernizzi T, Bottoli A, Autelitano A. Effect of timolol, latanoprost, and dorzolamide on circadian IOP in glaucoma or ocular hypertension. Invest Ophthalmol Vis Sci. 2000 Aug;41(9):2566-73.

4. Nakamoto K, Yasuda N. Effect of concomitant use of latanoprost and brinzolamide on 24-hour variation of IOP in normal-tension glaucoma. J Glaucoma. 2007 Jun-Jul;16 (4):352-7.
5. Konstas AG, Topouzis F, Leliopoulou O, et al. 24-hour intraocular pressure control with maximum medical therapy compared with surgery in patients with advanced open-angle glaucoma. Ophthalmology. 2006 May;113(5): 761-5.

6. Nakakura S, Nomura Y, Ataka S, Shiraki K. Relation between office intraocular pressure and 24-hour intraocular pressure in patients with primary open-angle glaucoma treated with a combination of topical antiglaucoma eye drops. J Glaucoma. 2007 Mar;16(2):201-4.

7. Itoh $\mathrm{Y}, \mathrm{Nakamoto} \mathrm{K}$, Horiguchi $\mathrm{H}$, et al. Twenty-fourhour variation of intraocular pressure in primary openangle glaucoma treated with triple eye drops. J Ophthalmol. 2017;2017:4398494.

8. Kiddee W, Atthavuttisilp S. The effects of selective laser trabeculoplasty and travoprost on circadian intraocular pressure fluctuations: A randomized clinical trial. Medicine (Baltimore). $2017 \mathrm{Feb}$;96(6):e6047.

9. Lee AC, Mosaed S, Weinreb RN, Kripke DF, Liu JH. Effect of laser trabeculoplasty on nocturnal intraocular pressure in medically treated glaucoma patients. Ophthalmology. 2007 Apr;114(4):666-70.

10. Matsuoka M, Ando A, Minamino K, et al. Dampening of diurnal intraocular pressure fluctuation by combined trabeculotomy and sinusotomy in eyes with open-angle glaucoma. J Glaucoma. 2013 Apr-May;22(4):290-3.

11. Gulati V, Fan S, Zhao M, Maslonka MA, Gangahar C, Toris $\mathrm{CB}$. Diurnal and nocturnal variations in aqueous humor dynamics of patients with ocular hypertension undergoing medical therapy. Arch Ophthalmol. 2012 Jun;130 (6):677-84.

12. Fan S, Agrawal A, Gulati V, Neely DG, Toris CB. Daytime and nighttime effects of brimonidine on IOP and aqueous humor dynamics in participants with ocular hypertension. J Glaucoma. 2014 Jun-Jul;23(5):276-81.

13. Quaranta L, Biagioli E, Riva I, et al. Effect of trabeculectomy and canaloplasty on intraocular pressure modifications after postural changes in open-angle glaucoma. Acta Ophthalmol. 2014 Sep;92(6):e498-9.

14. Fukuda T, Nakamoto K, Yasuda N, Furusawa C. Maitomaishin $C$ heiyo senichutai setsujojutsugo no ganatsu nichinai hendo [Effects of trabeculectomy with mitomycin $\mathrm{C}$ on 24-hour intraocular pressure variation]. Rinsho Ganka [Jpn J Clin Opthalmol]. 2006 Dec;60(12):1961-3. Japanese.

(Received, November 8, 2020)

(Accepted, December 15, 2020)

Journal of Nippon Medical School has adopted the Creative Commons Attribution-NonCommercial-NoDerivatives 4.0 International License (https://creativecommons.org/licenses/by-nc-nd/4.0/) for this article. The Medical Association of Nippon Medical School remains the copyright holder of all articles. Anyone may download, reuse, copy, reprint, or distribute articles for non-profit purposes under this license, on condition that the authors of the articles are properly credited. 